Research Paper

\title{
Adjuvant Chemotherapy Correlates with Improved Survival after Radical Cystectomy in Patients with pT3b (Macroscopic Perivesical Tissue Invasion) Bladder Cancer
}

Hyung Suk Kim ${ }^{1 *}$, Songzhe Piao ${ }^{1,3^{*}}$, Kyung Chul Moon², Chang Wook Jeong1, Cheol Kwak ${ }^{1}$, Hyeon Hoe $\mathrm{Kim}^{1}$, Ja Hyeon $\mathrm{Ku}^{1} \bowtie$

1. Department of Urology, Seoul National University College of Medicine, Seoul, Korea

2. Department of Pathology, Seoul National University College of Medicine, Seoul, Korea

3. Department of urology, Yanbian University Hospital, Yanji, Jilin Province, China

* Hyung Suk Kim and Songzhe Piao contributed equally to this work as a co-first author.

$\triangle$ Corresponding author: Ja Hyeon Ku, M.D. PhD., Department of Urology, Seoul National University Hospital, 101 Daehak-ro, Jongno-gu, Seoul 110-744, Korea. Tel: 82-2-2072-0361; Fax: 82-2-742-4665; E-mail: kuuro70@snu.ac.kr

() 2015 Ivyspring International Publisher. Reproduction is permitted for personal, noncommercial use, provided that the article is in whole, unmodified, and properly cited. See http:/ /ivyspring.com/terms for terms and conditions.

Received: 2015.03.27; Accepted: 2015.05.07; Published: 2015.06.29

\begin{abstract}
Object: To elucidate the impact of adjuvant chemotherapy $(\mathrm{ACH})$ on survival in patients with urothelial carcinoma of the bladder (UCB) diagnosed with pT3 disease (perivesical tissue invasion).

Methods: We reviewed the clinical data of 424 patients who underwent radical cystectomy (RC) with bilateral pelvic lymphadenectomy for UCB in our institution between 1991 and 2012. None of the patients received neoadjuvant chemotherapy. Of all patients, 101 (23.8\%) were diagnosed with PT3 disease: PT3a in 43 patients and pT3b in 58 patients. The Kaplan-Meier method with the log-rank test was used to estimate and compare overall survival (OS) and cancer-specific survival (CSS) between groups. Multivariate Cox proportional hazard models were used to identify predictors of OS and CSS.

Results: Five-year OS (48.5\% vs. $45.6 \%$ ) and CSS (56.1\% vs. $56.2 \%)$ were similar in the pT3a and pT3b groups ( $\mathrm{p}=0.658$ and 0.840 , respectively). In all $\mathrm{pT} 3$ patients, $\mathrm{ACH}$ administration was an independent predictor for OS $(p=0.018)$, but not CSS $(p=0.623)$ on multivariate analyses. On multivariate analysis according to the $\mathrm{PT} 3$ sub-stage, $\mathrm{ACH}$ was significantly associated with improved OS (hazard ratio [HR] 0.35; 95\% confidence interval $[\mathrm{Cl}] 0.17-0.72, \mathrm{P}=0.004$ ) and CSS (HR 0.33; 95\% Cl 0.10-0.85, $\mathrm{p}=0.022$ ) in only the $\mathrm{pT} 3 \mathrm{~b}$ group.

Conclusion: Our data suggest that in $\mathrm{PT} 3 \mathrm{~b}$ disease, characterized by macroscopic perivesical tissue invasion, patients may obtain an OS benefit from the administration of $\mathrm{ACH}$. Thus, our findings provide evidence for establishing appropriate indications for $\mathrm{ACH}$ in muscle invasive $\mathrm{UCB}$.
\end{abstract}

Key words: urothelial carcinoma; radical cystectomy; perivesical tissue invasion; adjuvant chemotherapy; survival

\section{Introduction}

Bladder cancer is the second most common urologic malignancy, with significant associated morbidity and mortality. ${ }^{1,2}$ The prognosis of patients with bladder cancer can be affected by a variety of fac- tors. ${ }^{3-5}$ Among these factors, local extension of the tumor is crucial in predicting the prognosis of non-metastatic muscle invasive bladder cancer. ${ }^{3-6}$ Depending on the depth of primary tumor invasion, 
the American Joint Committee on Cancer (AJCC) staging system classifies advanced bladder tumors as pT2, pT3, and pT4. Based on the 2002 AJCC TNM classification, tumors extending outside the bladder, but not into the adjacent organs, are categorized as having microscopic (pT3a) or macroscopic (pT3b) perivesical tissue invasion. The prognostic influence of this distinction of pT3 sub-stages on clinical outcomes, including recurrence and survival, has been evaluated in several studies, but conflicting results have been reported. ${ }^{7-11}$

Radical cystectomy (RC) with bilateral pelvic lymphadenectomy is a gold standard treatment for the local control of non-metastatic muscle invasive bladder cancer (pT2-T4aN0M0). ${ }^{2}$ However, a large number of bladder cancer patients develop recurrence and cancer-related death due to unrecognized occult nodal or distant metastasis at the time of RC.6,12 In light of this, to improve the survival of patients with locally advanced bladder cancer, the application of perioperative systemic chemotherapy combined with RC has been assessed by many investigators. ${ }^{13-17}$ There is adequate evidence of cisplatin-based neoadjuvant chemotherapy as a recommended therapeutic modality for advanced bladder cancer.13,15-17 However, the evidence supporting the utility of adjuvant chemotherapy $(\mathrm{ACH})$ in the management of advanced bladder cancer remains insufficient. ${ }^{14-18}$ Furthermore, as mentioned earlier, detailed classification of pT3 bladder cancers can make it more difficult to establish definite indications with regard to the use of $\mathrm{ACH}$.

In the current study, we sought to identify the clinical significance of sub-staging of pT3 urothelial carcinoma of the bladder (UCB) and assess the prognostic value of the administration of $\mathrm{ACH}$ in patients with pT3 urothelial carcinoma of the bladder (UCB).

\section{Materials and methods}

\section{Study population}

After receiving approval from the institutional review board of Seoul National University Hospital, we retrospectively reviewed the bladder cancer database of a single institution consisting of 424 patients who underwent RC with pelvic lymph node dissection (PLND) between January 1991 and December 2012. Patients with metastatic disease at the time of $\mathrm{RC}$, a pT2 or lower disease classification based on the final RC specimen, a history of neoadjuvant chemotherapy, and histology other than urothelial carcinoma were excluded from the study. Finally, a total of 101 patients who showed pT3 UCB at the final RC specimen were available for our final analysis. A portion of the patients included in this study was also included in previous our study. ${ }^{19}$

\section{Acquisition of data and definition of variables}

The pathologic stage and grade of all RC specimens were evaluated by a proficient genitourinary pathologist and were assigned according to the 2010 Tumor-Node-Metastasis classification of the 7th AJCC, and the 2004 World Health Organization system, respectively. Thus, the pathologic stage and grade of specimens from patients who had received surgery before the application of the updated guidelines were re-assessed. The pT3 stage was divided into pT3a or pT3b sub-stages according to the current guidelines. $^{2}$ pT3a disease was assigned to cases of only microscopic histological infiltration of the perivesical tissue without gross perivesical invasion. If perivesical invasion was visible and palpable on gross assessment of the RC specimen and could be confirmed on histological examination, pT3b disease was assigned. Other assessed covariates included clinical parameters such as age, gender, body mass index (BMI), American Society of Anesthesiologists score, clinical hydronephrosis, and adjuvant chemotherapy $(\mathrm{ACH})$ and pathologic parameters such as carcinoma in situ (CIS), lymphovascular invasion (LVI), perineural invasion (PNI), variant histology of urothelial carcinoma (UC), perivesical margin, lymph node (LN) status, and total number of removed LN. To facilitate the statistical analysis, age $(<60$ or $\geq 60$ years) and BMI $\left(<25\right.$ or $\left.\geq 25 \mathrm{~kg} / \mathrm{m}^{2}\right)$ were dichotomized. The LN status and clinical hydronephrosis were also analyzed as a binary variable according to their presence or absence. LVI was defined as the presence of tumor cells within an endothelium-lined space without underlying muscular walls. ${ }^{20} \mathrm{PNI}$ was defined as tumor invasion into the perineural sheath or endoneurium. ${ }^{21}$ The perivesical margin was only defined as the presence of tumor at inked soft tissue areas on the final pathologic slide of the RC specimen; therefore, the urethral or ureteral resection margin was not considered as a margin in this analysis. ACH was defined as that administered within 3 months postoperatively and consisted of cisplatin-based regimens including gemcitabine and cisplatin (GC) or methotrexate, vinblastine, adriamycin, and cisplatin (MVAC). In patients with significantly diminished renal function, carboplatin was administered with gemcitabine, replacing cisplatin.

\section{Follow-up}

Postoperative follow-up was conducted in accordance with institutional protocol. Patients were usually followed-up at least every 3-4 months for the first year, semiannually for the second year, and annually thereafter. Follow-up examinations consisted 
of a physical examination, blood test, chest radiography, kidney ultrasonography, and/or computed tomography urography. A bone scan was performed only in cases with bone-related symptoms. Cancer-specific survival (CSS) was defined as the interval from the time of RC to bladder cancer-related death. The duration of overall survival (OS) was estimated from the date of RC to the date of last follow-up or death from any cause. Data on living patients were censored from relevant analyses. Death was identified by reviewing medical charts and/or from the annual census of the Korea National Statistical Office. Cause of death was determined by the responsible physicians and from death certificates.

\section{Statistical analyses}

The pT3a and pT3b sub-stage groups were compared using the chi-square or Fisher's exact tests for categorical variables and the Mann-Whitney $U$ test for continuous variables. Continuous variables are expressed as the median and interquartile range (IQR); categorical variables are expressed as absolute numbers with relative percentages. Survival outcomes including OS and CSS were estimated using the Kaplan-Meier method and compared using a log-rank test in the entire patient cohort and sub-stage groups. Multivariate Cox proportional hazard models were used to assess the factors associated with sur- vival outcomes. All statistical analyses were conducted using SPSS software version 21.0 (SPSS Inc., Chicago, IL, USA); two-sided p-values of $<0.05$ were considered to be statistically significant.

\section{Results}

\section{Characteristics of the study cohort}

Table 1 summarizes the characteristics of the entire study cohort and the comparative results of various parameters between the pT3a and pT3b subgroups. The median age of the patients was 69.0 years (IQR: 63.0-75.5) and they were predominantly men $(85.1 \%)$. A majority of the patients $(98.0 \%)$ showed high-grade tumors on the pathologic examination of RC specimens. A total of 34 patients $(33.7 \%)$ were finally confirmed as having LN positive disease. All patients were divided into pT3 sub-stage groups: 43 $(42.6 \%)$ were diagnosed with pT3a (microscopic perivesical tissue invasion) and 58 (57.4\%) with pT3b (macroscopic perivesical tissue invasion) disease. Except for the total number of removed LN ( $p=0.048)$, there were no significant differences in the distribution of the other parameters evaluated, including pathologic tumor grade $(p=0.179)$, CIS $(p=0.093)$, LVI $(p=0.564)$, PNI $(p=0.549)$, LN status $(p=0.139)$ and the frequency of $\mathrm{ACH}(\mathrm{p}=0.228)$ between the pT3 sub-stage groups.

Table 1. Clinicopathologic parameters of the entire study cohort and comparative analysis results between pT3a and pT3b sub-stage groups.

\begin{tabular}{|c|c|c|c|c|}
\hline & $\begin{array}{l}\text { pT3 } \\
(n=101)\end{array}$ & $\begin{array}{l}\text { pT3a (microscopic) } \\
(\mathrm{n}=43)\end{array}$ & $\begin{array}{l}\text { pT3b(macroscopic) } \\
(\mathrm{n}=58)\end{array}$ & $\mathrm{p}$-value \\
\hline Age, year, median (IQR) & $69(63-75.5)$ & $69(63-76)$ & $69(60.5-74.25)$ & 0.479 \\
\hline$<60 \mathrm{yrs}$ & $20(19.8 \%)$ & $6(14.0 \%)$ & $14(24.1 \%)$ & 0.204 \\
\hline$\geq 60 \mathrm{yrs}$ & $81(80.2 \%)$ & $37(86.0 \%)$ & $44(75.9 \%)$ & \\
\hline \multicolumn{5}{|l|}{ Gender } \\
\hline Male & $86(85.1 \%)$ & $36(83.7 \%)$ & $50(86.2 \%)$ & 0.728 \\
\hline Female & $15(14.9 \%)$ & $7(16.3 \%)$ & $8(13.8 \%)$ & \\
\hline BMI(kg/m2), median (IQR) & $23.5(21.6-25.5)$ & $23.8(22.0-26.4)$ & $23.2(21.0-24.8)$ & 0.061 \\
\hline$<25$ & $73(72.3 \%)$ & $28(65.1 \%)$ & $45(77.6 \%)$ & 0.166 \\
\hline$\geq 25$ & $28(27.7 \%)$ & $15(34.9 \%)$ & $13(22.4 \%)$ & \\
\hline \multicolumn{5}{|l|}{ ASA score } \\
\hline 1 & $46(45.5 \%)$ & $18(41.9 \%)$ & $28(48.8 \%)$ & 0.741 \\
\hline 2 & $52(51.5 \%)$ & $24(55.8 \%)$ & $28(48.3 \%)$ & \\
\hline 3 & $3(3 \%)$ & $1(2.3 \%)$ & $2(3.4 \%)$ & \\
\hline \multicolumn{5}{|l|}{ Clinical hydronephrosis, $\mathbf{n}(\%)$} \\
\hline No & $72(71.3 \%)$ & $33(76.7 \%)$ & $39(67.2 \%)$ & 0.297 \\
\hline Yes & $29(28.7 \%)$ & $10(23.3 \%)$ & $19(32.8 \%)$ & \\
\hline \multicolumn{5}{|l|}{ Pathologic grade, n (\%) } \\
\hline Low grade & $2(2.0 \%)$ & $2(4.7 \%)$ & $0(0 \%)$ & 0.179 \\
\hline High grade & $99(98.0 \%)$ & $41(95.3 \%)$ & $58(100 \%)$ & \\
\hline \multicolumn{5}{|l|}{ CIS within bladder, $\mathrm{n}(\%)$} \\
\hline Absent & $91(90.1 \%)$ & $36(83.7 \%)$ & $55(94.8 \%)$ & 0.093 \\
\hline Present & $10(9.9 \%)$ & $7(16.3 \%)$ & $3(5.2 \%)$ & \\
\hline \multicolumn{5}{|l|}{ LVI within bladder, $\mathrm{n}(\%)$} \\
\hline Absent & $39(38.6 \%)$ & $18(41.9 \%)$ & $21(36.2 \%)$ & 0.564 \\
\hline Present & $62(61.4 \%)$ & $25(58.1 \%)$ & $37(63.8 \%)$ & \\
\hline \multicolumn{5}{|l|}{ Perivesical margin, $\mathbf{n}(\%)$} \\
\hline Absent & $95(94.1 \%)$ & $41(95.3 \%)$ & $54(93.1 \%)$ & 1.000 \\
\hline Present & $6(5.9 \%)$ & $2(4.7 \%)$ & $4(6.9 \%)$ & \\
\hline
\end{tabular}




\begin{tabular}{|c|c|c|c|c|}
\hline \multicolumn{5}{|l|}{ Perineural invasion, $\mathbf{n}(\%)$} \\
\hline Absent & $72(71.3 \%)$ & $32(74.4 \%)$ & $40(69.0 \%)$ & 0.549 \\
\hline Present & $29(28.7 \%)$ & $11(25.6 \%)$ & $18(31.0 \%)$ & \\
\hline \multicolumn{5}{|l|}{ Variant of UC, n (\%) } \\
\hline Absent & $85(84.2 \%)$ & $38(88.4 \%)$ & $47(81.0 \%)$ & 0.318 \\
\hline Present & $16(15.8 \%)$ & $5(11.6 \%)$ & $11(19.0 \%)$ & \\
\hline \multicolumn{5}{|l|}{ LN status, $n(\%)$} \\
\hline pN0 & $67(66.3 \%)$ & $32(74.4 \%)$ & $35(60.3 \%)$ & 0.139 \\
\hline $\mathrm{pN} \geq 1$ & $34(33.7 \%)$ & $11(25.6 \%)$ & $23(39.7 \%)$ & \\
\hline Total number of removed LNs (median, IQR) & $14(10-20)$ & $13(9-18)$ & $15.5(10.7-24.5)$ & 0.048 \\
\hline \multicolumn{5}{|l|}{ Adjuvant CTx, n (\%) } \\
\hline Not done & $47(46.5 \%)$ & $23(53.5 \%)$ & $24(41.4 \%)$ & 0.228 \\
\hline Done & $54(53.5 \%)$ & $20(46.5 \%)$ & $34(58.6 \%)$ & \\
\hline OS f/u duration (median, IQR) & $32.5(17.1-74.5)$ & $32.5(18.1-85.5)$ & $32.7(15.6-73.8)$ & 0.716 \\
\hline Alive & $49(48.5 \%)$ & $22(51.2 \%)$ & $27(46.6 \%)$ & 0.647 \\
\hline Death & $52(51.5 \%)$ & $21(48.8 \%)$ & $31(53.4 \%)$ & \\
\hline CSS f/u duration (median, IQR) & $32.5(17.1-74.5)$ & $32.5(18.1-85.5)$ & $32.7(15.6-73.8)$ & 0.731 \\
\hline Alive & $63(62.4 \%)$ & $27(62.8 \%)$ & $36(62.1 \%)$ & 0.941 \\
\hline Death & $38(37.6 \%)$ & $16(37.2 \%)$ & $22(37.9 \%)$ & \\
\hline
\end{tabular}

A

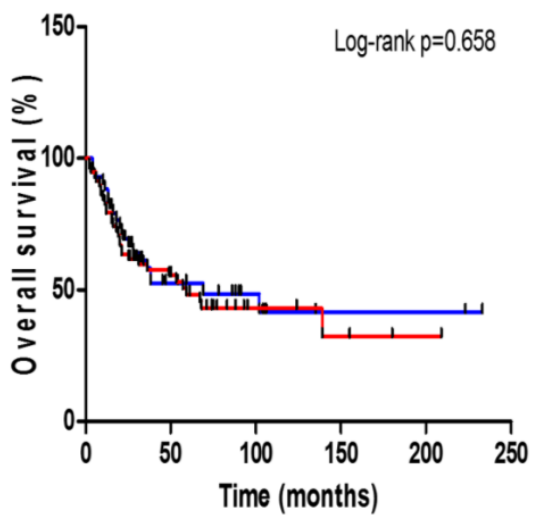

B

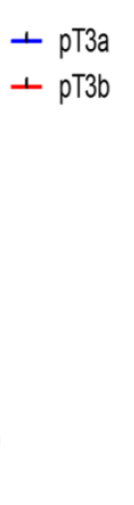

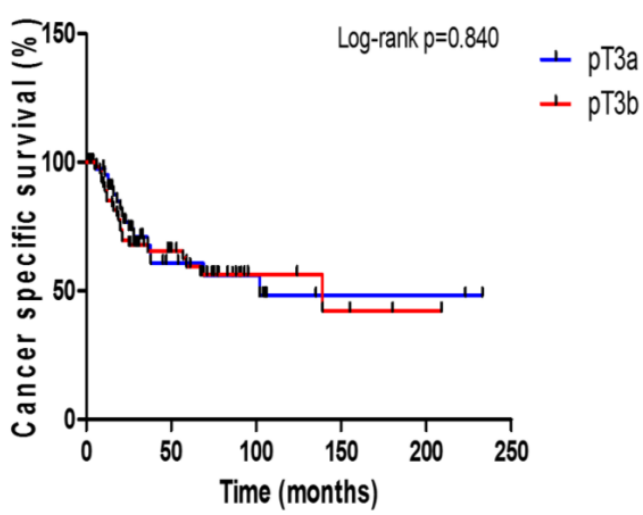

Figure 1. Kaplan-Meier plots for survival outcomes according to the $\mathrm{PT} 3$ sub-stage in all patients. (A) overall survival (B) cancer-specific survival

\section{Survival outcomes}

In this study cohort, a total of $52(51.5 \%)$ patients died of any cause with the median OS time of 18.6 months (IQR: 10.4-35.0 months); 38 (37.6\%) of these deaths were attributed to UCB. Follow-up durations were similar in the two pT3 sub-stage groups (Table 1). The 5-year OS and CSS rates were not significantly different between the pT3a and pT3b patients $(48.5 \%$ vs. $45.6 \%, p=0.658$, Fig. $1 \mathrm{~A} ; 56.1 \%$ vs. $56.2 \%$, $p=$ 0.840 , Fig. 1B). When comparing OS and CSS with respect to the $\mathrm{LN}$ status ( $\mathrm{pN} 0$ or $\mathrm{pN} \geq 1$ ), significant survival differences were not observed between the pT3 sub-stages in either the LN negative or positive group (Supplementary Fig. S1). The patients who received $\mathrm{ACH}$ had a better OS benefit than those who did not ( $p=0.025$, Fig. $2 \mathrm{~A})$, but ACH did not have an effect on CSS ( $p=0.635$, Fig. 2B).

When performing the subgroup analysis in each pT3 sub-stage group, there was a meaningful difference in OS ( $p=0.005$, Fig. $3 A)$, but not CSS $(p=0.148$, Fig. $3 \mathrm{~B})$, between those with and without $\mathrm{ACH}$ only in the pT3b group. However, there were no significant differences in OS and CSS according to the presence or absence of $\mathrm{ACH}$ in the pT3a group (Supplementary Fig. S2).

On multivariate analyses for the entire study cohort, the performance of $\mathrm{ACH}$ was the only independent predictor associated with improved OS (hazard ratio [HR]: 0.51; 95\% CI: 0.29-0.89; $p=0.018$ ), but not CSS ( $p=0.542)$, after controlling for the impact of other variables (Table 2). However, pT3 sub-staging was not an independent predictive factor of OS ( $\mathrm{p}=$ $0.421)$ or CSS $(p=0.964)$.

We also evaluated the variables associated with survival outcomes in each sub-stage group. When conducting the multivariate Cox regression analyses adjusting for other variables in each group, we could not identify any factors that showed significant correlation with survival outcomes in the pT3a group. However, in the pT3b group, the performance of $\mathrm{ACH}$ was independently correlated with improved OS (HR: 0.35; 95\% CI: 0.17-0.72; $\mathrm{p}=0.004$ ) and CSS (HR 0.33; 95\% CI 0.10-0.85, $\mathrm{p}=0.022$ ) (Table 3 ). 
Table 2. Multivariable Cox regression analyses for evaluating variables predicting overall survival and cancer specific survival in the entire study cohort (pT3)

\begin{tabular}{|c|c|c|c|c|c|c|}
\hline & \multicolumn{3}{|c|}{ Overall survival } & \multicolumn{3}{|c|}{ Cancer specific survival } \\
\hline & Adjusted HR & $95 \% \mathrm{CI}$ & $\mathrm{p}$-value & Adjusted HR & $95 \% \mathrm{CI}$ & p-value \\
\hline \multicolumn{7}{|l|}{ Age } \\
\hline$<60 \mathrm{yrs}$ & Reference & & & Reference & & \\
\hline$\geq 60 \mathrm{yrs}$ & 1.22 & $0.52-2.87$ & 0.642 & 0.90 & $0.34-2.38$ & 0.835 \\
\hline \multicolumn{7}{|l|}{ Gender } \\
\hline Male & Reference & & & Reference & & \\
\hline Female & 1.44 & $0.64-3.20$ & 0.375 & 1.60 & $0.61-4.22$ & 0.338 \\
\hline \multicolumn{7}{|l|}{ BMI } \\
\hline$<25$ & Reference & & & Reference & & \\
\hline$\geq 25$ & 1.14 & $0.58-2.25$ & 0.699 & 0.90 & $0.39-2.06$ & 0.812 \\
\hline \multicolumn{7}{|l|}{ ASA score } \\
\hline 1 & Reference & & & Reference & & \\
\hline 2 & 1.38 & $0.72-2.66$ & 0.336 & 1.04 & $0.49-2.17$ & 0.925 \\
\hline 3 & 2.04 & $0.43-9.75$ & 0.371 & 1.11 & $0.13-9.49$ & 0.924 \\
\hline \multicolumn{7}{|l|}{ Clinical hydronephrosis } \\
\hline Absent & Reference & & & Reference & & \\
\hline Present & 0.96 & $0.46-1.99$ & 0.914 & 1.20 & $0.53-2.76$ & 0.659 \\
\hline \multicolumn{7}{|l|}{ pT3 sub-stage } \\
\hline pT3a & Reference & & & Reference & & \\
\hline pT3b & 1.38 & $0.69-2.74$ & 0.352 & 0.99 & $0.45-2.18$ & 0.989 \\
\hline \multicolumn{7}{|l|}{ Pathologic grade } \\
\hline Low & Reference & & & & & \\
\hline High & 0.76 & $0.09-6.79$ & 0.809 & & & \\
\hline \multicolumn{7}{|l|}{ CIS } \\
\hline Absent & Reference & & & Reference & & \\
\hline Present & 0.98 & $0.30-3.13$ & 0.969 & 0.24 & $0.03-1.99$ & 0.187 \\
\hline \multicolumn{7}{|l|}{ LVI } \\
\hline Absent & Reference & & & Reference & & \\
\hline Present & 1.68 & $0.94-3.03$ & 0.082 & 1.91 & $0.82-4.45$ & 0.136 \\
\hline \multicolumn{7}{|l|}{ Perivesical margin } \\
\hline Negative & Reference & & & Reference & & \\
\hline Positive & 1.75 & $0.63-4.84$ & 0.284 & 1.66 & $0.52-5.22$ & 0.389 \\
\hline \multicolumn{7}{|l|}{ Perineural invasion } \\
\hline Absent & Reference & & & Reference & & \\
\hline Present & 1.14 & $0.56-2.30$ & 0.722 & 0.91 & $0.39-2.10$ & 0.821 \\
\hline \multicolumn{7}{|l|}{ Variant of UC } \\
\hline Absent & Reference & & & Reference & & \\
\hline Present & 1.25 & $0.54-2.89$ & 0.610 & 1.11 & $0.39-3.18$ & 0.842 \\
\hline \multicolumn{7}{|l|}{ LN status } \\
\hline Negative & Reference & & & Reference & & \\
\hline Positive & 1.04 & $0.51-2.09$ & 0.921 & 1.06 & $0.46-2.45$ & 0.886 \\
\hline Total number of removed LNs & 0.98 & $0.95-1.01$ & 0.229 & 0.97 & $0.94-1.01$ & 0.126 \\
\hline \multicolumn{7}{|l|}{ Adjuvant CTx, n (\%) } \\
\hline Not done & Reference & & & Reference & & \\
\hline Done & 0.51 & $0.29-0.89$ & 0.018 & 0.79 & $0.37-1.69$ & 0.542 \\
\hline
\end{tabular}

A

B
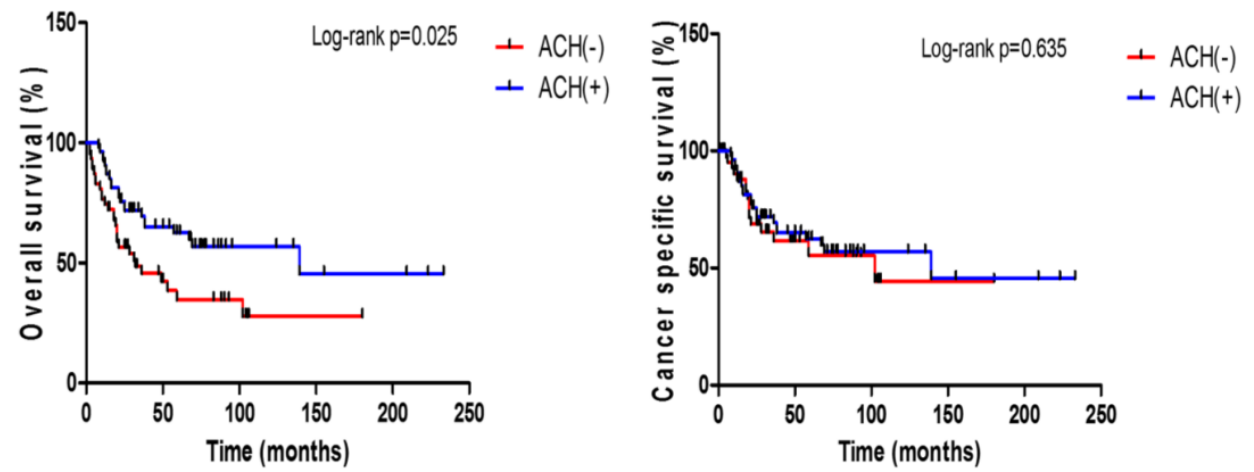

Figure 2. Kaplan-Meier plots for survival outcomes according to the presence or absence of adjuvant chemotherapy in all patients. (A) overall survival (B) cancer-specific survival 
A

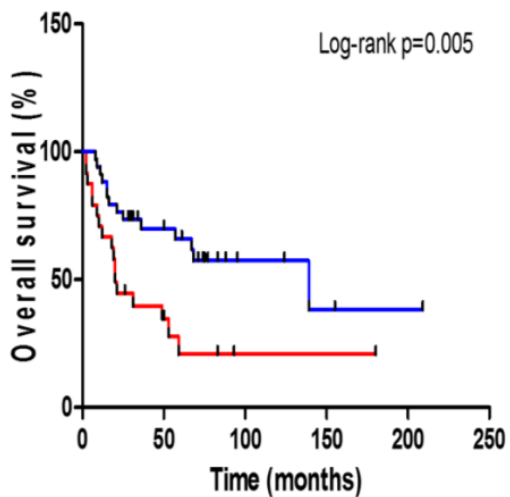

B

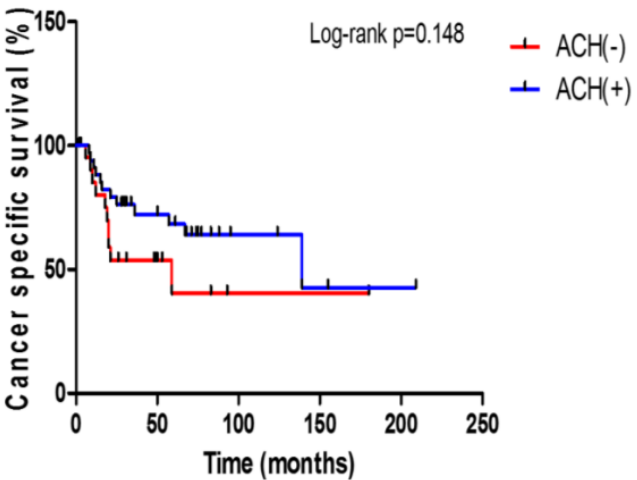

Figure 3. Kaplan-Meier plots for survival outcomes according to the presence or absence of adjuvant chemotherapy in the pT3b subgroup. (A) overall survival (B) cancer-specific survival

Table 3. Multivariable Cox regression analyses for evaluating variables predicting overall survival and cancer specific survival in patients with $\mathrm{PT} 3 \mathrm{~b}$ disease

\begin{tabular}{|c|c|c|c|c|c|c|}
\hline & \multicolumn{3}{|c|}{ Overall survival } & \multicolumn{3}{|c|}{ Cancer specific survival } \\
\hline & Adjusted HR & $95 \% \mathrm{CI}$ & $\mathrm{p}$-value & Adjusted HR & $95 \% \mathrm{CI}$ & $\mathrm{p}$-value \\
\hline \multicolumn{7}{|l|}{ Age } \\
\hline$<60 \mathrm{yrs}$ & Reference & & & Reference & & \\
\hline$\geq 60 \mathrm{yrs}$ & 1.00 & $0.31-3.19$ & 0.998 & 1.74 & $0.37-8.24$ & 0.485 \\
\hline \multicolumn{7}{|l|}{ Gender } \\
\hline Male & Reference & & & Reference & & \\
\hline Female & 1.62 & $0.47-5.53$ & 0.442 & 3.22 & $0.59-17.51$ & 0.175 \\
\hline \multicolumn{7}{|l|}{ BMI } \\
\hline$<25$ & Reference & & & Reference & & \\
\hline$\geq 25$ & 1.33 & $0.52-3.40$ & 0.556 & 0.74 & $0.21-2.57$ & 0.635 \\
\hline \multicolumn{7}{|l|}{ ASA score } \\
\hline 1 & Reference & & & Reference & & \\
\hline 2 & 2.34 & $0.86-6.34$ & 0.096 & 1.29 & $0.37-4.47$ & 0.687 \\
\hline 3 & 1.94 & $0.19-19.74$ & 0.574 & 2.03 & $0.17-23.97$ & 0.573 \\
\hline \multicolumn{7}{|l|}{ Clinical hydronephrosis } \\
\hline Absent & Reference & & & Reference & & \\
\hline Present & 0.83 & $0.33-2.08$ & 0.694 & 0.92 & $0.30-2.81$ & 0.889 \\
\hline \multicolumn{7}{|l|}{ CIS } \\
\hline Absent & Reference & & & Reference & & \\
\hline Present & 4.48 & $0.66-30.25$ & 0.124 & 2.38 & $0.16-35.26$ & 0.530 \\
\hline \multicolumn{7}{|l|}{ LVI } \\
\hline Absent & Reference & & & Reference & & \\
\hline Present & 1.90 & $0.86-4.21$ & 0.111 & 4.19 & $1.39-12.58$ & 0.011 \\
\hline \multicolumn{7}{|l|}{ Perivesical margin } \\
\hline Negative & Reference & & & Reference & & \\
\hline Positive & 2.71 & $0.59-12.37$ & 0.197 & 2.65 & $0.44-15.85$ & 0.287 \\
\hline \multicolumn{7}{|l|}{ Perineural invasion } \\
\hline Absent & Reference & & & Reference & & \\
\hline Present & 0.55 & $0.21-1.44$ & 0.224 & 0.32 & $0.10-1.01$ & 0.052 \\
\hline \multicolumn{7}{|l|}{ Variant of UC } \\
\hline Absent & Reference & & & Reference & & \\
\hline Present & 1.94 & $0.60-6.25$ & 0.264 & 1.07 & $0.24-4.79$ & 0.925 \\
\hline \multicolumn{7}{|l|}{ LN status } \\
\hline Negative & Reference & & & Reference & & \\
\hline Positive & 0.99 & $0.38-2.57$ & 0.984 & 0.37 & $0.10-1.33$ & 0.128 \\
\hline Total number of removed LNs & 0.97 & 0.93-1.01 & 0.188 & 0.97 & $0.92-1.02$ & 0.307 \\
\hline \multicolumn{7}{|l|}{ Adjuvant CTx, n (\%) } \\
\hline Not done & Reference & & & Reference & & \\
\hline Done & 0.35 & $0.17-0.72$ & 0.004 & 0.33 & $0.10-0.85$ & 0.022 \\
\hline
\end{tabular}




\section{Discussion}

Since 2002, the AJCC TNM classification of bladder cancer has divided the pT3 (perivesical tissue invasion) disease into pT3a (microscopic) and pT3b (macroscopic) sub-stages. Even though perivesical tissue invasion is a known adverse prognostic factor, a definite conclusion regarding the prognostic implication of this distinction of pT3 disease has not been reached thus far. ${ }^{7-11}$

Several reports have demonstrated the prognostic significance of pT3 sub-staging. In a cohort of 2,388 pT2b-pT3b bladder cancer patients obtained from the SEER database, Scosyrev et al. reported that pT3a was associated with poorer OS than $\mathrm{pT} 2 \mathrm{~b}$ (deep muscle invasion) irrespective of LN status, but the OS difference between $\mathrm{pT} 3 \mathrm{~b}$ and $\mathrm{pT} 3 \mathrm{a}$ was only observed in node-positive disease. ${ }^{11}$ In a multicenter study consisting of 808 patients with pT3 disease, Tilki et al. reported that $\mathrm{pT} 3 \mathrm{~b}$ disease showed worse 5-year recurrence free survival (RFS) and CSS rates than pT3a disease in only LN negative UCB. ${ }^{8}$ Furthermore, patients with pT3b disease had significantly higher frequency of LN invasion and administration of $\mathrm{ACH}$ than those with pT3a disease. Similarly, Neuzillet et al. demonstrated that patients with pT2b and pT3a disease showed comparable survival outcomes, which were better than those of patients with pT3b disease in only cases of pN0 disease. ${ }^{9}$ The frequency of LN metastasis and administration of $\mathrm{ACH}$ was significantly greater in the pT3b group than in the pT3a group. Moreover, these investigators suggested the benefit of $\mathrm{ACH}$ in patients with pT3bN0 tumors, given that the metastasis-free survival differences between pT2b-pT3a and pT3b were not significant for $\mathrm{LN}$ negative patients who had received $\mathrm{ACH}$.

Contrary to the studies mentioned above, in 75 patients with LN negative pT3 disease, consisting of 46 pT3a and 29 pT3b patients, Boudreaux et al. identified no significant differences in survival outcomes, such as OS, CSS, and RFS, between the pT3 subgroups. ${ }^{7}$ Likewise, in a similar scale retrospective study including 74 patients with pT3N0 ( 35 pT3a and 39 pT3b) disease, Dincel et al. found that pT3b did not have worse survival outcomes (OS and CSS) than pT3a disease. ${ }^{10}$

In the current study, we could identify no significant differences in OS and CSS depending on pT3 sub-staging. When performing survival analyses according to LN status, the survival differences between pT3a and pT3b were also not observed in LN positive or LN negative patients. These findings correspond to previous study results. ${ }^{7,10} \mathrm{In}$ addition, the distribution of LN status and frequency of $\mathrm{ACH}$ were not significantly different between the two pT3 sub-staging groups, which was not the case in previous studies. 8,9 In summary, the prognostic impact of pT3 sub-classification on survival outcomes after RC was not confirmed in our study.

$\mathrm{ACH}$ is commonly administered for the management of locally advanced bladder cancer. In particular, bladder cancer showing perivesical tissue invasion on final pathologic examination after $R C$ has been generally considered as a possible indication for ACH. ${ }^{14,22-24}$ However, unlike neoadjuvant chemotherapy, which is currently the established modality for improving survival on the basis of several meta-analysis and prospective studies, ${ }^{13,15-17}$ evidence supporting the utility and indication of chemotherapy in an adjuvant setting is currently lacking. This is because a well-designed prospective study can be difficult given the side effects of chemotherapy, the poor postoperative general condition of patients, and diminished renal function.14-18 Furthermore, as mentioned earlier, the debates regarding the prognostic significance of pT3 sub-staging may make it more difficult to establish the definite indications for the use of $\mathrm{ACH}$.

In our study, although there were no meaningful survival differences between the pT3 subgroups, an OS benefit related to the administration of $\mathrm{ACH}$ was observed in the entire pT3 patient group. On subgroup analysis according to the pT3 sub-stage, an OS benefit of $\mathrm{ACH}$ was confirmed only in the pT3b sub-stage. Furthermore, on multivariate Cox regression analyses adjusting for the impact of other covariates in the $\mathrm{pT} 3 \mathrm{~b}$ group, the administration of $\mathrm{ACH}$ was an independent predictor of better OS and CSS. OS is widely used as the primary endpoint to assess the clinical outcome of oncologic treatment, ${ }^{25}$ and the final goal of bladder cancer treatment is to prolong the survival of patients. Therefore, we cautiously suggest that $\mathrm{pT} 3 \mathrm{~b} \mathrm{UCB}$ may be a potential indication for $\mathrm{ACH}$ to improve postoperative survival. This suggestion also corresponds to the findings of the study by Neuzillet et al. ${ }^{9}$

There are several limitations to the current study. First, our study is limited owing to its retrospective design with inherent selection bias. A potential bias may exist because patients treated with neoadjuvant chemotherapy were excluded from the study. Our data were extracted from patients treated with RC by multiple surgeons over a 20 -year period and, therefore, may not represent current clinical practice. Several time-dependent variables, such as surgical technique (i.e., PLND template) and pathologic report content (i.e., variant histology) may have impacted our results. Furthermore, inter-surgeon variation regarding surgery related expertise, determination of $\mathrm{ACH}$ implementation, type of $\mathrm{ACH}$ reg- 
imen assigned, and postoperative follow-up strategies could have affected the current study results. Finally, our study cohort was relatively small, with patients recruited from a single institution. Therefore, the results drawn from this study should be further verified through well-designed prospective and randomized clinical trials.

In conclusion, in this study, the detailed classification of pT3 disease according to the current AJCC staging system had no effect on survival outcomes after RC. However, the administration of $\mathrm{ACH}$ in pT3b disease showed a close correlation with improvement of postoperative survival. Therefore, we suggest that $\mathrm{pT} 3 \mathrm{~b}$ disease should be considered as a potential indication for chemotherapy in an adjuvant setting. Further verification through well-designed prospective and randomized clinical trials will be required to establish macroscopic perivesical invasion as an indication for $\mathrm{ACH}$ in $\mathrm{UCB}$.

\section{Supplementary Material}

Fig.S1 - Fig.S2.

http://www.jcancer.org/v06p0750s1.pdf

\section{Acknowledgements}

\section{Ethical approval of studies}

This study design and the use of patients' information stored in the hospital database were approved by the Institutional Review Board (IRB) at the Seoul National University Hospital. The approval number is H-1502-070-648. We were given exemption from getting informed consents by the IRB because the present study is a retrospective study and personal identifiers were completely removed and the data were analyzed anonymously. Our study was conducted according to the ethical standards laid down in the 1964 Declaration of Helsinki and its later amendments.

\section{Financial support}

There were no grants or other external funding sources for this study.

\section{Authors' contribution}

Conception and design: Ja Hyeon $\mathrm{Ku}$, Chang Wook Jeong, Cheol Kwak, Hyeon Hoe Kim

Data acquisition: Hyung Suk Kim, Songzhe Piao, Ja Hyeon Ku, Kyung Chul Moon

Data analysis and interpretation: Hyung Suk Kim, Songzhe Piao

Drafting the manuscript: Hyung Suk Kim, Songzhe Piao

Critical revision of the manuscript for scientific and factual content: Hyung Suk Kim, Ja Hyeon Ku

Statistical analysis: Hyung Suk Kim, Songzhe
Piao

Supervision: Ja Hyeon $\mathrm{Ku}$, Chang Wook Jeong, Cheol Kwak, Hyeon Hoe Kim

(Both Hyung Suk Kim and Songzhe Piao contributed equally to this work as a co-first author.)

\section{Competing Interests}

The authors have declared that no competing interest exists.

\section{References}

1. Burger M, Catto JWF, Dalbagni G, et al. Epidemiology and risk factors of urothelial bladder cancer. Eur Urol. 2013;63:234-241.

2. Witjes JA, Comperat E, Cowan NC, et al. EAU guidelines on muscle-invasive and metastatic bladder cancer: summary of the 2013 guidelines. Eur Urol 2014;65:778-792.

3. Bassi P, Ferrante GD, Piazza N, et al. Prognostic factors of outcome after radical cystectomy for bladder cancer: a retrospective study of a homogeneous patient cohort. J Urol. 1999;161:1494-1497.

4. Frazier HA, Robertson JE, Dodge RK, Paulson DF. The value of pathologic factors in predicting cancer-specific survival among patients treated with radical cystectomy for transitional cell carcinoma of the bladder and prostate. Cancer. 1993;71:3993-4001.

5. Herr HW, Faulkner JR, Grossman HB, et al. Surgical factors influence bladder cancer outcomes: a cooperative group report. J Clin Oncol. 2004;22:2781-2789.

6. Stein JP, Lieskovsky G, Cote R, et al. Radical cystectomy in the treatment of invasive bladder cancer: long-term results in 1,054 patients. J Clin Oncol. 2001;19:666-675.

7. Boudreaux KJ Jr., Chang SS, Lowrance WT, et al. Comparison of American Joint Committee on Cancer pathologic stage T3a versus T3b urothelial carcinoma: analysis of patient outcomes. Cancer. 2009;115:770-775.

8. Tilki D, Svatek RS, Karakiewicz PI, et al. pT3 Substaging is a prognostic indicator for lymph node negative urothelial carcinoma of the bladder. J Urol. 2010;184:470-474.

9. Neuzillet $\mathrm{Y}$, Lebret $\mathrm{T}$, Molinie $\mathrm{V}$, et al. Perivesical fat invasion in bladder cancer: implications for prognosis comparing pT2b, pT3a and pT3b stages and consequences for adjuvant chemotherapy indications. BJU Int. 2012;110:1736-1741.

10. Dincel C, Kara C, Balci U, et al. Comparison of microscopic (pT3a) and gross extravesical extension (pT3b) in pathological staging of bladder cancer: analysis of patient outcomes. Int Urol Nephrol. 2013;45:387-393.

11. Scosyrev E, Yao J, Messing E. Microscopic invasion of perivesical fat by urothelial carcinoma: implications for prognosis and pathology practice. Urology. 2010;76:908-913.

12. Karl A, Carroll PR, Gschwend JE, et al. The Impact of Lymphadenectomy and Lymph Node Metastasis on the Outcomes of Radical Cystectomy for Bladder Cancer. Eur Urol. 2009;55:826-835.

13. Grossman HB, Natale RB, Tangen CM, et al. Neoadjuvant chemotherapy plus cystectomy compared with cystectomy alone for locally advanced bladder cancer. N Eng J Med. 2003;349:859-866.

14. Cognetti F, Ruggeri EM, Felici A, et al. Adjuvant chemotherapy with cisplatin and gemcitabine versus chemotherapy at relapse in patients with muscle-invasive bladder cancer submitted to radical cystectomy: an Italian, multicenter, randomized phase III trial. Ann Oncol. 2012;23:695-700.

15. Meeks JJ, Bellmunt J, Bochner BH, et al. A systematic review of neoadjuvant and adjuvant chemotherapy for muscle-invasive bladder cancer. Eur Urol. 2012;62:523-533.

16. Sternberg CN, Bellmunt J, Sonpavde G, et al. ICUD-EAU International Consultation on Bladder Cancer 2012: chemotherapy for urothelial carcinoma-neoadjuvant and adjuvant settings. Eur Urol. 2013;63:58-66.

17. Booth CM, Siemens DR, Li G, et al. Perioperative chemotherapy for muscle-invasive bladder cancer: a population-based outcomes study. Cancer. 2014;120:1630-1638.

18. Raghavan D, Bawtinhimer A, Mahoney J, Eckrich S, Riggs S. Adjuvant chemotherapy for bladder cancer-why does level 1 evidence not support it? Ann Oncol. 2014;25:1930-1934.

19. Kim HS, Moon KC, Jeong CW, Kwak C, Kim HH, Ku JH. The clinical significance of intraoperative ureteral frozen section analysis at radical cystectomy for urothelial carcinoma of the bladder. World J Urol. 2015:33:359-366.

20. Bolenz C, Herrmann E, Bastian PJ, et al. Lymphovascular invasion is an independent predictor of oncological outcomes in patients with lymph node-negative urothelial bladder cancer treated by radical cystectomy: a multicentre validation trial. BJU Int. 2010;106:493-499.

21. Hong SK, Kwak C, Jeon HG, Lee E, Lee SE. Do vascular, lymphatic, and perineural invasion have prognostic implications for bladder cancer after radical cystectomy? Urology. 2005;65:697-702.

22. Park J, Park S, Song C, et al. Effectiveness of adjuvant chemotherapy in transitional cell carcinoma of the urinary bladder with lymph node involvement 
and/or lymphovascular invasion treated by radical cystectomy. Urology. 2007;70:257-262.

23. Leow JJ, Martin-Doyle W, Rajagopal PS, et al. Adjuvant chemotherapy for invasive bladder cancer: a 2013 updated systematic review and meta-analysis of randomized trials. Eur Urol. 2014;66:42-54.

24. Yelfimov DA, Frank I, Booriian SA, Thapa P, Cheville JC, Tollefson MK, Adjuvant chemotherapy is associated with decreased mortality after radical cystectomy for locally advanced bladder cancer. World J Urol. 2014;32:1463-1468.

25. Driscoll JJ, Rixe O. Overall survival: still the gold standard: why overall survival remains the definitive end point in cancer clinical trials. The Cancer Journal. 2009;15:401-405. 